\title{
Minimal Flavor Protection: A New Flavor Paradigm in Warped Models
}

\author{
José Santiagd* \\ Institute for Theoretical Physics, ETH, CH-8093, Zürich, Switzerland
}

\begin{abstract}
We propose a new flavor paradigm for models with warped extra dimensions. The idea is to impose the minimal amount of flavor protection to make warped models compatible with all current flavor and electroweak precision constraints. We discuss a particular realization of this minimal flavor protection in the quark sector, by means of a flavor symmetry acting on the right handed down sector. Hierarchical quark masses and mixing angles are naturally reproduced through wave function localization, and flavor violating processes are predicted, in the absence of large brane kinetic terms for the right handed down quarks, below but not too far from current experimental limits in several channels. With this new flavor pattern, models with warped extra dimensions can be accessible through direct production of new resonances at the LHC and also through precision flavor experiments.
\end{abstract}

\section{INTRODUCTION}

Models with warped extra dimensions [1] provide a neat explanation to the vast hierarchy between the Planck and electroweak scales, with the added bonus of a very appealing structure of flavor. First, the exponential localization of the chiral zero modes of bulk fermions [2] naturally predicts a hierarchical structure of quark masses and mixing angles [3, 4. Even more important, the effect of the warp factor on the localization of the gauge boson Kaluza-Klein (KK) modes provides a very special flavor protection, dubbed the RS-GIM mechanism [3, 5, 6. Thanks to this mechanism, flavor violating processes are suppressed by small mixing angles or quark masses. Thus, flavor violating processes involving light fermions, which are very constrained experimentally, are naturally small. In most of the studies of models with warped extra dimensions, it has been implicitly assumed that, either flavor physics is safe, thanks to the RS-GIM mechanism, or that possible flavor violations above experimental limits can be fixed (with some fine-tuning or with appropriate flavor symmetries) without altering the natural generation of hierarchical quark masses and mixing angles and without affecting flavor diagonal physics in a sensitive way. With that assumption, realistic models with a low scale of new physics have been recently constructed [7], using ideas like custodial symmetry [8] and a protection of the $Z b_{L} \bar{b}_{L}$ coupling [9, 10]. The result is that KK excitations of gauge bosons as light as $M_{K K} \gtrsim 3 \mathrm{TeV}$ can be compatible with electroweak precision tests (EWPT) and a natural explanation of the fermion mass hierarchy.

Detailed analyses of flavor constraints [5, 6, 11, 12, 13, show that the RS-GIM mechanism is in fact extremely effective (see also [14), predicting flavor violating processes close to, but below current experimental bounds in almost all channels. The only notable exception is CP violation in the Kaon system, $\epsilon_{K}$, which, if simultaneous flavor violating left handed $(\mathrm{LH})$ and right handed $(\mathrm{RH})$ currents mediated by the gluon KK modes are present, requires the mass of the lightest KK mode to be (depending on the particular model) [12]

$$
M_{K K} \gtrsim 20-30 \mathrm{TeV}, \quad \text { (from } \epsilon_{K}, \text { LR contribution). }
$$

The main goal of this note is to emphasize that, despite recent claims that models with warped extra dimensions suffer from a serious flavor problem, the above discussion shows that the situation is actually much better than that. As we just said, the RS-GIM mechanism is strikingly effective, seriously missing only in one observable and due to a very particular chirality enhanced contribution. Our proposal is to make use of the already good flavor properties of models with warped extra dimensions and introduce only the minimal amount of flavor protection to suppress the dangerous contribution to $\epsilon_{K}$, without sizably modifying the remaining flavor properties of the model. ${ }^{1}$ This minimal flavor protection (MFP) prescription can be realized, for instance, by imposing a $U(3)$ flavor symmetry under which all fields are singlets except for the three fields that give rise to the charge $-1 / 3 \mathrm{RH}$ quark zero modes (and other fields related to them by the symmetries of the model), which transform as a triplet. The symmetry is only broken by brane localized Yukawa couplings (or by localized mass or kinetic mixing for the multiplets of the

\footnotetext{
*Electronic address: santiago@itp.phys.ethz.ch

${ }^{1}$ Less minimal proposals of Havor protection in models with warped extra dimensions have been presented in [15]. See [16] for a model that is compatible with flavor constraints at the expense of not fully explaining the hierarchy problem.
} 
brane gauge symmetry that do not contain the $d_{R}$ zero modes) generating in this way non-trivial quark masses in the down sector. As we will see in detail below, this new paradigm still allows for the quark masses and mixing angles to be induced by wave function localization. The masses in the up sector are generated by double hierarchies, whereas the ones in the down sector are generated by a single hierarchy and therefore are naturally less spread. This provides a rationale for the smaller hierarchy of quark masses in the down sector $\left(m_{d} / m_{b} \sim 10^{-3}\right)$ than in the up sector $\left(m_{u} / m_{t} \sim 10^{-5}\right)$. Also, LH flavor violating currents mediated by the gluon KK modes are predicted as in the standard realization of flavor (below but not too far from current experimental bounds) and therefore could be measured in the near future, particularly observables in the $B$ system [17]. The plan of the paper is the following: In the next section we describe in detail a particular realization of the MFP proposal in the quark sector, discussing the absence of the leading contribution to $\Delta F=2$ left-right (LR) processes from dimension 6 operators. In section III we discuss sub-leading (higher dimension or loop suppressed operators) flavor violating contributions which give the main constraint in MFP models. We discuss the numerical significance of the corresponding bounds in section IV and finally conclude with a discussion of the results and an outlook of future prospects in section $\mathrm{V}$.

\section{MINIMAL FLAVOR PROTECTION IN THE QUARK SECTOR}

In this section we describe in detail an explicit realization of MFP in the quark sector. For the sake of the presentation we consider a model with a fundamental Higgs localized at the IR brane and discuss the results for other models in section IV. The discussion closely follows the one in [12. The background is a slice of $\mathrm{AdS}_{5}$,

$$
d s^{2}=\left(\frac{R}{z}\right)^{2}\left(\eta_{\mu \nu} d x^{\mu} d x^{\nu}-d z^{2}\right)
$$

with $R \leq z \leq R^{\prime} . R \approx M_{\mathrm{Pl}}^{-1}$ and $R^{\prime} \approx \mathrm{TeV}^{-1}$ are the position of the UV and IR branes, respectively. In order to make it compatible with a low KK scale we assume a bulk $S U(3) \times S U(2)_{L} \times S U(2)_{R} \times U(1)_{X}$ gauge group 8 and a discrete $L \leftrightarrow R$ symmetry that exchanges the $S U(2)_{L}$ and $S U(2)_{R}$ groups, to protect the $Z \bar{b}_{L} b_{L}$ coupling $[9$. We introduce three families of five-dimensional bulk quarks with the following quantum numbers under $S U(2)_{L} \times S U(2)_{R} \times U(1)_{X}$,

$$
Q^{i}=(2,2)_{2 / 3}, \quad Q^{u i}=(1,1)_{2 / 3}, \quad Q^{d i}=(1,3)_{2 / 3}, \quad \tilde{Q}^{d i}=(3,1)_{2 / 3},
$$

where $i=1,2,3$ is the flavor index. The choice of boundary conditions is

$$
Q^{i}=\left(\begin{array}{c}
\chi^{i}=\left(\begin{array}{c}
\chi^{u i} \\
\chi^{d i}
\end{array}\right)[-+] \\
q^{i}=\left(\begin{array}{l}
q^{u i} \\
q^{d i}
\end{array}\right)[++]
\end{array}\right), \quad Q^{u i}=U^{i}[--], \quad Q^{d i}=\left(\begin{array}{c}
X^{i}[+-] \\
U^{\prime i}[+-] \\
D^{i}[--]
\end{array}\right), \quad \tilde{Q}^{d i}=\left(\begin{array}{c}
\tilde{X}^{i}[+-] \\
\tilde{U}^{\prime i}[+-] \\
\tilde{D}^{i}[+-]
\end{array}\right),
$$

where $\chi^{i}$ and $q^{i}$ are $S U(2)_{L}$ doublets with hypercharge, $7 / 6$ and $1 / 6, \tilde{Q}^{d i}$ forms a triplet under $S U(2)_{L}$ and $X^{i}, U^{\prime i}$ and $D^{i}$ are the three components of the $S U(2)_{R}$ triplet, with $T_{R}^{3}=+1,0,-1$, respectively. - $(+)$ denotes Dirichlet boundary condition for the LH $(\mathrm{RH})$ chirality of the bulk fermion at the corresponding brane (the first sign is for the UV brane and the second for the IR brane). A LH $(\mathrm{RH})$ fermion zero mode corresponds to a field with $[++]([--])$ boundary conditions. With our choice of boundary conditions, $q_{L}^{i}, U_{R}^{i}$ and $D_{R}^{i}$ are the only fields that have zero modes. The multiplet $\tilde{Q}^{d i}$ has been included to ensure that the heavy physics is approximately invariant under the discrete $L \leftrightarrow R$ symmetry [9]. In order to realize our MFP prescription, we assume a global $U(3)$ symmetry under which $Q^{d i}$ and $\tilde{Q}^{d i}$ transform as triplets and all the other fields transform as singlets.

Bulk fermions admit a bulk mass term that can always be taken to be diagonal in flavor space. Using standard notation we parametrize these mass terms as

$$
\left(\frac{R}{z}\right)^{4}\left[\frac{c_{q i}}{z} \bar{Q}^{i} Q^{i}+\frac{c_{u i}}{z} \bar{Q}^{u i} Q^{u i}+\frac{c_{d}}{z}\left(\bar{Q}^{d i} Q^{d i}+\overline{\tilde{Q}}^{d i} \tilde{Q}^{d i}\right)\right]
$$

where we have explicitly written a common bulk mass for all $Q^{d i}$ and $\tilde{Q}^{i d}$ as imposed by the $U(3)$ flavor symmetry and the discrete $L \leftrightarrow R$ symmetry. These bulk fields admit zero modes given by

$$
\begin{aligned}
q_{L}^{i}(x, z) & =\chi_{c_{q i}}(z) q_{L}^{(0) i}(x)+\ldots \\
U_{R}^{i}(x, z) & =\chi_{-c_{u i}}(z) u_{R}^{(0) i}(x)+\ldots \\
D_{R}^{i}(x, z) & =\chi_{-c_{d}}(z) d_{R}^{(0) i}(x)+\ldots
\end{aligned}
$$


with wave function that results in canonical normalization of the four-dimensional fields given by

$$
\chi_{c}(z) \equiv \frac{1}{\sqrt{R^{\prime}}}\left(\frac{z}{R}\right)^{2}\left(\frac{z}{R^{\prime}}\right)^{-c} f_{c}
$$

with

$$
f_{c} \equiv \sqrt{\frac{1-2 c}{1-\left(\frac{R^{\prime}}{R}\right)^{2 c-1}}}
$$

The fermion zero mode masses come from $U(3)$ violating IR localized Yukawa couplings. After EWSB, they read,

$$
\begin{aligned}
\mathcal{L}_{y} & =-\frac{v}{\sqrt{2}}\left(\frac{R}{R^{\prime}}\right)^{4} R^{\prime}\left[\left(\bar{q}^{u i}-\bar{\chi}^{d i}\right) \tilde{Y}_{i j}^{u} U^{j}+\bar{q}^{d i} \tilde{Y}_{i j}^{d}\left(D^{j}+\tilde{D}^{j}\right)+\text { h.c. }+\ldots\right] \\
& =-\frac{v}{\sqrt{2}}\left(\bar{u}_{L}^{(0) i} f_{q i} \tilde{Y}_{i j}^{u} f_{-u j} u_{R}^{(0) j}+\bar{d}_{L}^{(0) i} f_{q i} \tilde{Y}_{i j}^{d} f_{-d} d_{R}^{(0) j}\right)+\text { h.c. }+\ldots .
\end{aligned}
$$

We have written the dimensionful five-dimensional Yukawa couplings as $Y_{5 D}^{u, d}=R^{\prime} \tilde{Y}^{u, d}$, where the (now dimensionless) Yukawa couplings $\tilde{Y}^{u, d}$ are assumed to be anarchic $3 \times 3$ matrices (all entries order one and order one determinant). In the second line we have explicitly written the effective four-dimensional Yukawa couplings for the quark zero modes. $v=246 \mathrm{GeV}$ is the warped down Higgs vev. The hierarchical structure of the quark masses and mixing angles is then explained by a hierarchical structure of the $f_{q i}$ and $f_{-u i}[5]$ (recall that the $U(3)$ flavor symmetry forces a common $\left.f_{-d}\right)$, which are generated by non-hierarchical five-dimensional bulk masses, see Eq. (10),

$$
f_{q 1} \ll f_{q 2} \ll f_{q 3}, \quad f_{-u 1} \ll f_{-u 2} \ll f_{-u 3} .
$$

The SM quark masses can then be diagonalized with unitary rotations,

$$
\begin{aligned}
& \frac{v}{\sqrt{2}}\left(\mathcal{U}_{L}^{\dagger} f_{q} \tilde{Y}_{u} f_{-u} \mathcal{U}_{R}\right)_{i j}=m_{i}^{u} \delta_{i j} \\
& \frac{v}{\sqrt{2}}\left(\mathcal{D}_{L}^{\dagger} f_{q} \tilde{Y}_{d} f_{-d} \mathcal{D}_{R}\right)_{i j}=m_{i}^{d} \delta_{i j}
\end{aligned}
$$

which, due to the hierarchical structure of $f_{q,-u}$, are hierarchical in the case of $\mathcal{U}_{L, R}$ and $\mathcal{D}_{L}$,

$$
\left|\left(\mathcal{U}_{L}\right)_{i j}\right| \sim\left|\left(\mathcal{D}_{L}\right)_{i j}\right| \sim \frac{f_{q i}}{f_{q j}}, \quad\left|\left(\mathcal{U}_{R}\right)_{i j}\right| \sim \frac{f_{-u i}}{f_{-u j}}, \quad i \leq j,
$$

and therefore the CKM matrix is also hierarchical,

$$
\left|\left(V_{C K M}\right)_{i j}\right|=\left|\left(\mathcal{U}_{L}^{\dagger} \mathcal{D}_{L}\right)_{i j}\right| \sim \frac{f_{q i}}{f_{q j}}, \quad i \leq j .
$$

From here on, the $\sim$ symbol means that the equalities are true up to Yukawa dependent order one numbers. On the other hand, $\mathcal{D}_{R}$ is the order one unitary matrix that diagonalizes the matrix

$$
\left(\tilde{Y}^{d}\right)_{i k}^{\dagger} f_{q k}^{2} \tilde{Y}_{k j}^{d}
$$

The diagonal masses are then also hierarchical,

$$
m_{i}^{u} \sim \frac{v}{\sqrt{2}} f_{q i} f_{-u i}, \quad m_{i}^{d} \sim \frac{v}{\sqrt{2}} f_{q i} f_{-d} .
$$

Assuming that the hierarchical pattern of quark masses and mixing angles comes from wave function localization and not due to hierarchies in the fundamental Yukawa couplings (as we have just discussed above), the CKM matrix and the up type quark masses approximately fix the values of the following localization parameters,

$$
\begin{aligned}
f_{q 3} & \sim 1, \quad f_{q 2} \sim \lambda^{2}, \quad f_{q 1} \sim \lambda^{3}, \\
f_{-u 3} & \sim 1, \quad f_{-u 2} \sim \frac{m_{c}}{m_{t}} \lambda^{-2}, \quad f_{-u 1} \sim \frac{m_{u}}{m_{t}} \lambda^{-3}
\end{aligned}
$$


where $\lambda \sim 0.22$ is the Cabbibo angle. The localization parameter in the RH down sector is common to all three flavors, giving a ratio of masses

$$
m_{d} / m_{s} / m_{b} \approx[(0.03-0.1) / 1 /(50-70)]^{\exp } \sim[0.22 / 1 / 21]^{\mathrm{MFP}},
$$

where the first set of numbers is the experimental ratios of masses (with the variation indicating the uncertainty) at the scale of new physics $\sim 3 \mathrm{TeV}$ and the second set is the ratio of masses we obtain (as usual up to order one Yukawa couplings) from Eq. (18) and the values of $f_{q i}$ from Eq. (19). Note that, thanks to the smaller mass hierarchy in the down sector, the numerical differences can be easily accounted for by a mild hierarchy in the order one Yukawa couplings. This could not have been achieved with universality of either $f_{q}$ or $f_{-u}$, since the hierarchy in the up sector is too large to be accounted for without hierarchical Yukawa couplings in that case.

We can now turn to the flavor violation in this model. In the current eigenstate basis, in which Yukawa couplings are non-diagonal, the coupling of the quark zero modes to the gauge boson KK modes, after integration over the extra dimension, is diagonal but flavor dependent, except for $d_{R}^{(0) i}$, for which the $U(3)$ symmetry guarantees flavor independence,

$$
\bar{u}_{L}^{(0) i} g_{q i}^{(n)} G^{(n)} u_{L}^{(0) i}+\bar{u}_{R}^{(0) i} g_{u i}^{(n)} G^{(n)} u_{R}^{(0) i}+\bar{d}_{L}^{(0) i} g_{q i}^{(n)} G^{(n)} d_{L}^{(0) i}+\bar{d}_{R}^{(0) i} g_{d}^{(n)} G^{(n)} d_{R}^{(0) i},
$$

where we have written the coupling to KK gluons, $G_{\mu}^{(n)} \equiv T^{a} G_{\mu}^{(n) a}$, which is the largest one. The physical basis, with diagonal quark masses, is obtained by the unitary rotations in Eq. (14). The flavor dependence of the couplings to the KK gluons induce flavor changing neutral currents (FCNC) upon such rotations [18], with couplings

$$
\begin{aligned}
& \left(g_{u L}^{(n)}\right)_{i j}=g_{q k}^{(n)}\left(\mathcal{U}_{L}\right)_{k i}^{*}\left(\mathcal{U}_{L}\right)_{k j}, \quad\left(g_{d L}^{(n)}\right)_{i j}=g_{q k}^{(n)}\left(\mathcal{D}_{L}\right)_{k i}^{*}\left(\mathcal{D}_{L}\right)_{k j}, \\
& \left(g_{u R}^{(n)}\right)_{i j}=g_{u k}^{(n)}\left(\mathcal{U}_{R}\right)_{k i}^{*}\left(\mathcal{U}_{R}\right)_{k j}, \quad\left(g_{d R}^{(n)}\right)_{i j}=g_{d}^{(n)} \delta_{i j} .
\end{aligned}
$$

Our MFP prescription guarantees flavor independence of the couplings of the RH down quarks to the gauge boson KK modes, and therefore no flavor violating RH currents are generated in the down sector (due to gauge KK mode exchange).

Although the effect of the full gluon KK tower can be easily taken into account [12, 13, it is instructive to look at the couplings to the first KK mode to understand the RS-GIM mechanism in action. The coupling to the first gluon KK mode is given, to a very good approximation, by [12]

$$
g_{x} \approx g_{s *}\left(-\frac{1}{\log R^{\prime} / R}+f_{x}^{2} \gamma\left(c_{x}\right)\right),
$$

where here $x$ denotes the corresponding $q i,-u i$ or $-d, g_{s *} \approx 6$ is the bulk QCD gauge coupling (the actual fourdimensional coupling is, in the absence of large brane kinetic terms, $\left.g_{s *} / \sqrt{\log \left(R^{\prime} / R\right)}\right)$ and $\gamma(c)$ is an order one function. The first term is flavor universal and does not induce any FCNC. The second, however is flavor dependent and generates FCNC as shown in Eq. (23). Inserting the rotation matrices, Eq. (15), we obtain

$$
\left(g_{u L, d L}^{(1)}\right)_{i j} \sim g_{s *} f_{q i} f_{q j}, \quad\left(g_{u R}^{(1)}\right)_{i j} \sim g_{s *} f_{-u i} f_{-u j}, \quad\left(g_{d R}^{(1)}\right)_{i j}=g_{d}^{(1)} \delta_{i j} .
$$

This shows the RS-GIM and the MFP mechanisms at work. The left handed FCNC are suppressed by ratios of the CKM entries, see Eq. (19), whereas the up type right handed FCNC are suppressed by ratios of the light to heavy quark masses, Eq. 200. Finally, the RH down type FCNC are absent due to the flavor symmetry. Although we have exemplified this effect with the coupling to the first gluon KK mode, the protection extends to all the modes and to all gauge bosons. In the above discussion, we have neglected brane localized operators. Brane localized mass terms, for instance, can violate the MFP prescription, as they can mix the $d_{R}^{(0) i}$ with the heavy $q^{(n) i}$, which have family dependent couplings to the gauge boson KK modes. Thus, if the fermion quantum numbers of the fields allow for such mixing, the flavor symmetry should remain unbroken, on the corresponding brane, for the multiplets of the surviving symmetry in that brane that contain the $d_{R}^{(0) i}$. Brane kinetic terms [19] for $Q^{d i}$, on the other hand, cannot be forbidden by the flavor symmetry, as it is explicitly broken at the brane for these fields. In particular, they induce non-universal couplings of the $d_{R}^{(0) i}$ to the KK gluons at the IR brane, which in turn, generate FCNC in the right handed down sector in the physical basis. This effect can be however parametrically suppressed by a loop factor and will be discussed in detail in section III.

The flavor violating couplings to the gauge boson KK modes induce flavor violating four-fermion interactions, once the heavy fields are integrated out. We take as an example the $\Delta S=2$ contribution to $\Delta m_{K}$ and $\epsilon_{K}$, although all other $\Delta F=2$ processes are similar. The relevant operator has the following structure,

$$
\frac{1}{2 M_{K K}^{2}}\left[\left(\left(g_{d L}^{(1)}\right)_{d s} \bar{d}_{L} T^{a} \gamma^{\mu} s_{L}+\left(g_{d R}^{(1)}\right)_{d s} \bar{d}_{R} T^{a} \gamma^{\mu} s_{R}\right)\left(\left(g_{d L}^{(1)}\right)_{d s} \bar{d}_{L} T^{a} \gamma_{\mu} s_{L}+\left(g_{d R}^{(1)}\right)_{d s} \bar{d}_{R} T^{a} \gamma_{\mu} s_{R}\right)\right]
$$


where $T^{a}$ are the color matrices in the fundamental representation, $d_{L, R}$ and $s_{L, R}$ denote the physical down and strange quarks and we have included the first gluon KK mode (with mass denoted by $M_{K K}$ ) for illustration. These dimension 6 operators can be put in the standard basis by using Fierz identities and properties of the color matrices to get a Hamiltonian,

$$
\mathcal{H}^{\Delta S=2}=C_{1}^{s d} Q_{1}^{s d}+\tilde{C}_{1}^{s d} \tilde{Q}_{1}^{s d}+C_{4}^{s d} Q_{4}^{s d}+C_{5}^{s d} Q_{5}^{s d}
$$

where the coefficients read,

$$
\begin{aligned}
C_{1}^{s d} & =\frac{1}{6} \frac{1}{M_{K K}^{2}}\left(g_{d L}^{(1)}\right)_{d s}^{2}, & \tilde{C}_{1}^{s d} & =\frac{1}{6} \frac{1}{M_{K K}^{2}}\left(g_{d R}^{(1)}\right)_{d s}^{2}, \\
C_{4}^{s d} & =-\frac{1}{M_{K K}^{2}}\left(g_{d L}^{(1)}\right)_{d s}\left(g_{d R}^{(1)}\right)_{d s}, & C_{5}^{s d} & =\frac{1}{3} \frac{1}{M_{K K}^{2}}\left(g_{d L}^{(1)}\right)_{d s}\left(g_{d R}^{(1)}\right)_{d s}
\end{aligned}
$$

and we have used standard notation for the operators ( $\alpha$ and $\beta$ are color indices)

$$
\begin{array}{ll}
Q_{1}^{s d}=\bar{d}_{L}^{\alpha} \gamma^{\mu} s_{L}^{\alpha} \bar{d}_{L}^{\beta} \gamma^{\mu} s_{L}^{\beta}, & \tilde{Q}_{1}^{s d}=\bar{d}_{R}^{\alpha} \gamma^{\mu} s_{R}^{\alpha} \bar{d}_{R}^{\beta} \gamma^{\mu} s_{R}^{\beta} \\
Q_{4}^{s d}=\bar{d}_{R}^{\alpha} s_{L}^{\alpha} \bar{d}_{L}^{\beta} s_{R}^{\beta}, & Q_{5}^{s d}=\bar{d}_{R}^{\alpha} s_{L}^{\beta} \bar{d}_{L}^{\beta} s_{R}^{\alpha} .
\end{array}
$$

Our MFP prescription ensures that the coefficients of the operators that involve $\mathrm{RH}$ currents, $\tilde{Q}_{1}^{s d}, Q_{4}^{s d}$ and $Q_{5}^{s d}$, are zero at this order.

Using the results of [20], it was shown in Ref. [12] that the RS-GIM mechanism is enough to suppress almost all flavor violating observables below current experimental limits (but not too far below, in particular in observables in the $B$ system). The only observable that gives a constraint on the KK excitation of the gauge bosons stronger than the one obtained from EWPT is the measurement of CP violation in the Kaon system, $\epsilon_{K}$. Furthermore, the constraint is significant only if there are simultaneous flavor violations in both left and right currents. The reason is that the effect of $\Delta S=2$ operators with both chiralities has an enhancement as compared with the ones that only involve one chirality proportional to

$$
\frac{3}{4}\left(\frac{m_{K}}{m_{s}\left(\mu_{L}\right)+m_{d}\left(\mu_{L}\right)}\right)^{2} \eta_{1}^{-5} \approx 140
$$

where $\eta_{1}$ comes from the RGE running. Therefore only the (imaginary parts of) the coefficients $C_{4,5}^{s d}$ are strongly constrained. Our MFP prescription guarantees, in the absence of large BKT, the vanishing of FCNC in RH down currents and therefore that $\tilde{C}_{1}^{s d}=C_{4,5}^{s d}=0$. Once the LR contributions are absent, all the other observables are typically less constraining than EWPT.

\section{EXTRA SOURCES OF FLAVOR VIOLATION}

In the previous section we have seen how, in the absence of BKTs, our MFP prescription prevents the appearance of FCNC RH currents in the down sector, from dimension 6 operators generated by the exchange of gauge boson KK modes. Then, the only flavor observable which induces a strong experimental constraint does not receive the LR chirality enhanced contribution that makes it dangerous. Let us now discuss other sources of FCNC that are present in our MFP model. Although they are all formally sub-leading, as they correspond to dimension 8 operators or are loop suppressed, the absence of the leading contribution makes them the main source of flavor violation and therefore we have to consider their effect. We discuss them in turn.

\section{A. Mass Mixing with Kaluza-Klein Modes}

These new effects can have two different origins. The first is the mixing of the fermion zero modes with their (vector-like) KK excitations, which induces in general FCNC for the $Z$ boson and for any gauge boson KK mode (including the KK gluons). The second is the mixing of the $Z$ with its KK excitations, if they had FCNC couplings to the fermion zero modes. This latter effect does not occur for the RH down quark zero modes as, due to flavor universality, they do not have FCNC with the $Z$ KK modes. Furthermore, the corrections to the LH currents, although a priori flavor violating, have a very effective suppression due to the mechanism in 9 and we have checked that they are indeed negligibly small. The effect of the vector-like excitations of the quarks on the couplings to the $Z$ boson have 
been computed for models with warped extra dimensions in [21], using the general results of [22]. The outcome is that flavor violating couplings of the $Z$ to the $\mathrm{RH}$ down quarks are suppressed by the RS-GIM mechanism while couplings to the LH down quarks are forbidden by the MFP mechanism. The reason is that the dimension 6 contribution from the mixing with vector-like quarks comes from the effects of doublets $\left(q^{(n)}\right)$ for RH currents and singlets $\left(d^{(n)}\right)$ for LH currents and therefore the latter are the ones that the MFP mechanism protects. ${ }^{2}$ Thus, the relevant constraints from dimension 8 operators are expected to come from the exchange of KK gluons, which we consider now.

The Yukawa mixing of $d_{R}^{(0) i}$ with the KK excitations of $q^{i}$ induce flavor violations in the coupling of $d_{R}^{(0) i}$ to the gluon KK modes. Using the mass insertion approximation (in the numerical scans that we discuss below we have included exactly the effect of fermion mixing by numerically diagonalizing the corresponding mass matrices), we obtain, for the coupling of $d_{R}^{(0) i}$ to the first KK gluon

$$
\left(g_{d R}^{(1)}\right)_{i j} \approx g_{s *} f_{-d}^{2}\left[\mathcal{D}_{R}^{\dagger}\left(\tilde{Y}^{d}\right)^{\dagger} f_{q}^{(1)}\left(\frac{v}{\sqrt{2} M_{q(1)}}\right)^{2} \tilde{Y}^{d} \mathcal{D}_{R}\right]_{i j} \sim g_{s *} f_{-d}^{2}\left(\frac{v}{\sqrt{2} M_{K K}}\right)^{2},
$$

where we have included for illustration only the first fermion KK mode, $q^{(1) i}$, with a mass denoted in matrix form by $M_{q(1)}$ and we have denoted with $f_{q}^{(1)}$ the diagonal matrix containing the (order one) couplings of the $q^{(1) i}$ to the first gluon KK mode. In the last equality we have assumed that the fermion KK modes have masses similar to the ones of the gluon KK modes. (In our numerical scans we have not made use of that assumption but have included the exact mass of the fermion KK modes.) Thus, after EWSB, we do have a LR contribution to $\epsilon_{K}$ with coefficient,

$$
C_{4}^{s d(\mathrm{MFP})} \sim \frac{g_{s *}^{2}}{M_{K K}^{2}}\left(\frac{v}{\sqrt{2} M_{K K}}\right)^{2} f_{q 1} f_{q 2} f_{-d}^{2}
$$

to be compared with the one that appears in the standard realization of flavor in models with warped extra dimensions

$$
C_{4}^{s d(\mathrm{RS})} \sim \frac{g_{s *}^{2}}{M_{K K}^{2}} f_{q 1} f_{q 2} f_{-d 1} f_{-d 2} .
$$

Thus, there is a relative suppression with respect to the standard result of order

$$
\frac{C_{4}^{s d(\mathrm{MFP})}}{C_{4}^{s d(\mathrm{RS})}} \sim\left(\frac{v}{\sqrt{2} M_{K K}}\right)^{2} \frac{f_{-d}}{f_{-d 1}}=\left\{\begin{array}{l}
2.5 \times 10^{-2}\left(\frac{3 \mathrm{TeV}}{M_{K K}}\right)^{2} \frac{f_{-d} / f_{-d 1}}{0.22 / 0.03}, \\
7 . \times 10^{-3}\left(\frac{3 \mathrm{TeV}}{M_{K K}}\right)^{2} \frac{f_{-d} / f_{-d 1}}{0.22 / 0.1}
\end{array}\right.
$$

where, for the numerical estimates, we have assumed that the masses of the first fermion KK modes are approximately flavor independent $\sim 3 \mathrm{TeV}$ and we have taken the values of the localization parameters from Eq. (21). In the usual realization of flavor in models with warped extra dimensions, the typical value of $C_{4}^{s d(\mathrm{RS})}$ is about two orders of magnitude too large to be compatible with experimental data [12. Thus, the suppression factor that we obtain seems to be just of the right order of magnitude to make models with MFP and a low scale of new physics compatible with flavor constraints. This suppression assumes that the KK excitations of $q^{i}$ are at least as massive as the KK gluons. This is true for $[++]$ or $[--]$ boundary conditions but if the corresponding fermions have twisted $([+-]$ or $[-+])$ boundary conditions, ultralight modes could appear. If these ultralight modes mix sizably with the RH down quark zero modes, they can induce too large $\mathrm{CP}$ violation in the Kaon system. It should be kept in mind, however, that although KK quarks much lighter than the gauge boson KK modes are expected in models of natural EWSB [7, they are usually related to the top and do not necessarily mix significantly with the $\mathrm{RH}$ down sector. Furthermore, since the leading contribution in MFP models comes from a dimension 8 operator, the effect decouples like $C_{4}^{s d(M F P)} \sim M_{K K}^{-4}$, as opposed to models without such protection, for which the decoupling goes like $C_{4}^{s d(R S)} \sim M_{K K}^{-2}$. In order to better assess whether this suppression factor is enough or not, a more detailed numerical analysis is required. The result of such analysis is discussed in the next section.

\section{B. Effects of Brane Kinetic Terms}

Brane kinetic terms cannot be set to zero at all scales, as they are generated by quantum corrections [19. In particular, loops involving the brane localized Yukawa couplings will generate flavor non-universal brane kinetic terms

\footnotetext{
${ }^{2}$ The correction to the $Z \bar{d}_{L} s_{L}$ enters at dimension 6 but $\Delta S=2$ processes require two of these vertices and are therefore dimension 8 .
} 
for the RH down quarks. The relevant part of the Lagrangian can be written as

$$
\left(\frac{R}{z}\right)^{4}\left\{\left[\bar{Q}_{i}^{d}\left(\not D+\frac{c}{z}\right) Q_{i}^{d}\right]+\delta\left(z-R^{\prime}\right) R^{\prime}\left[\bar{Q}_{i}^{d} K_{i j} \not D Q_{j}^{d}-\frac{v}{\sqrt{2}}\left(\bar{q}^{d i} \tilde{Y}_{i j}^{d}\left[D^{j}+\tilde{D}^{j}\right]+\text { h.c. }\right)\right]\right\}+\ldots
$$

where $\not D$ is the (4d-slashed) covariant derivative (with all factors of the metric/vielbein stripped out but including the whole tower of gauge boson KK modes and therefore the full $z$ dependence) and $K_{i j}$ is the hermitian matrix parametrizing the parallel brane kinetic terms. ${ }^{3}$ The dots stand for other fields and $z$-dependent derivative terms (which are irrelevant for the discussion here). This matrix $K_{i j}$ is in fact non-calculable in 5D models, as it corresponds to a linearly divergent integral. It can be estimated, using NDA to be 12

$$
K \sim|\tilde{Y}|^{2} \frac{\Lambda R^{\prime}}{16 \pi^{2}}
$$

with $\Lambda$ the cut-off scale for the Yukawa interaction. The hermitian matrix $K$ can be diagonalized with a unitary rotation, $Q^{d i} \rightarrow \mathcal{U}_{i j} Q^{d j}$, such that

$$
\left(\mathcal{U}^{\dagger} K \mathcal{U}\right)_{i j}=a_{i} \delta_{i j}
$$

where $a_{i}$ are dimensionless numbers of the order of the $K$ entries. The Lagrangian has in the new basis the same form as the one above (in particular the bulk terms are not affected by this rotation, due to the MFP prescription) with diagonal BKT's and a new Yukawa coupling, still order one

$$
\tilde{Y}^{d} \rightarrow \mathcal{U}^{\dagger} \tilde{Y}^{d}
$$

which shows that we can, without loss of generality, start with diagonal (but in general family non-universal) BKTs.

The effect of diagonal fermion BKTs on the KK expansion was first studied in the last two references of [19. The result for the zero modes is that they maintain the same wave function profile as in the case of zero BKT and only the normalization is modified. The new normalization can be obtained from the previous one with the replacement

$$
f_{c} \rightarrow \frac{f_{c}}{\sqrt{1+a_{i} f_{c}^{2}}}
$$

In particular, the coupling to the first gauge boson KK mode, now reads, to a very good accuracy,

$$
g_{d i} \approx g_{s *}\left(-\frac{1}{\log R^{\prime} / R}+\left(\gamma\left(-c_{d}\right)+a_{i} \zeta\left(-c_{d}\right)\right) \frac{f_{-d}^{2}}{1+a_{i} f_{-d}^{2}}\right)=g_{d}^{\text {universal }}+g_{s *} \zeta\left(-c_{d}\right) a_{i} f_{-d}^{2}+\mathcal{O}\left(f_{-d}^{4}\right),
$$

where $\zeta(c)$ is an order one function of $c$ and in the second equality we have expanded in $f_{-d} \ll 1$. The large rotation in the $Q^{d}$ sector will then reintroduce the dangerous FCNC to the KK gluons in the RH down sector which are, a priori, only suppressed by the loop factor in $a_{i}$. Again a full numerical scan, that we preform in the next section, is required to asses whether that suppression is enough and whether the mild hierarchies that are required in the 5D Yukawas to reproduce the down sector masses, play a role in further suppressing these contributions.

\section{NUMERICAL SCANS}

The suppression provided by our MFP prescription on the LR contribution to CP violation in the Kaon system is just about right (in the absence of large BKT) to put $\epsilon_{K}$ around the current experimental limit. In order to be more quantitative about this bound, we have performed a numerical scan in which we have chosen different values of $0.4 \leq c_{q 3} \leq 0.45$, fixed the values of $c_{q 1, q 2}, c_{u i}$ and $c_{d}$ using Eqs. 119,21, and then generating random complex $3 \times 3$ matrices $\tilde{Y}^{u, d}$, satisfying $\left|\tilde{Y}^{u, d}\right| \leq 3$, in oder to keep perturbativity for the first few KK modes [12]. Out of the points generated, we have selected the ones for which the pattern of quark masses and mixing angles is similar to the experimentally observed (including the Jarlskog invariant) and with that subset we have computed the coefficients of the corresponding gluon mediated $\Delta F=2$ operators. In our numerical studies, we have not used the mass insertion

\footnotetext{
${ }^{3}$ We only need to consider parallel brane kinetic terms as classical renormalization of the singularities due to orthogonal brane kinetic terms bring them to the form of parallel brane kinetic terms [23].
} 


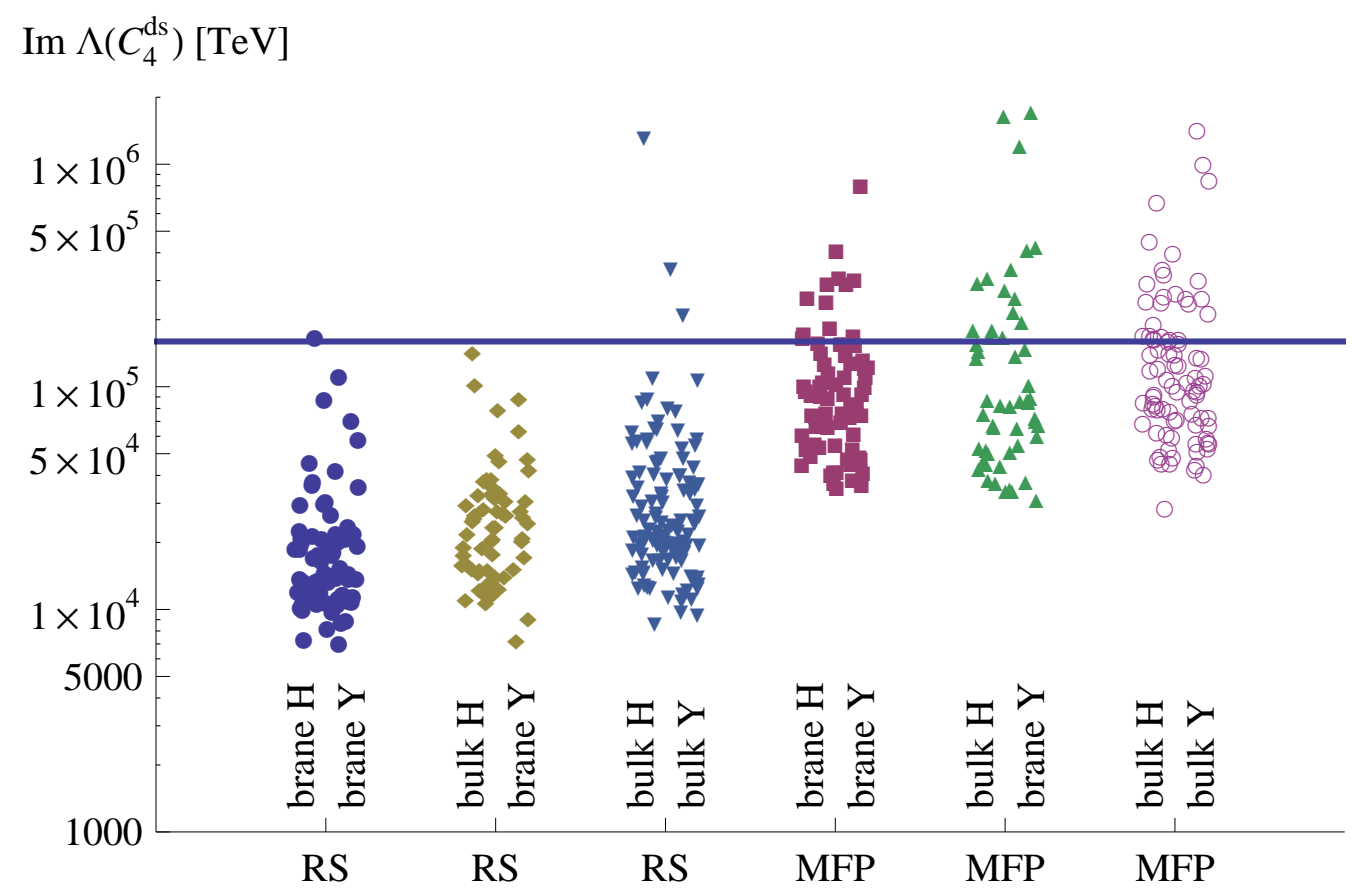

FIG. 1: Mass suppression of the imaginary part of the coefficient $C_{4}^{s d}$ in $\mathrm{TeV}$ for our three different models without (left three columns) and with MFP (right three columns). In all points we have fixed $M_{K K}=3 \mathrm{TeV}$ and the different sets correspond, from left to right, to standard RS with boundary Higgs, RS with bulk Higgs but boundary Yukawas, bulk Higgs with bulk up Yukawas and the same three models with MFP. The horizontal line corresponds to the experimental lower bound on the suppression scale. A random horizontal shift in the points has been introduced to facilitate visualization.

approximation, Eq. (33). In order to include the effect of fermion mixing to all orders we have numerically diagonalized the corresponding mass matrices (including the KK modes of all fermions, not only $q^{(n) i}$ ) and computed the couplings to the gluon KK modes in the physical basis using the exact rotation matrices. In order to assess separately the two different effects discussed in the previous section we have not included fermion brane kinetic terms in these scans. They will be analyze in a more extensive scan described below. Also, for simplicity, we have not included BKT for the KK gluons. They can change the bound on the scale of new physics in either direction by about $50 \%$ in the case of models without MFP [12. If MFP is at work, the faster decoupling of the corresponding operators will imply a much smaller variation in the bound. In order to explore further different realizations of flavor, we have considered three different possibilities. The first one is the one we have discussed in the previous two sections, an IR brane localized Higgs. The other two possibilities involve a bulk Higgs, with profile in the extra dimension given by

$$
\phi(x, y)=\sqrt{\frac{2}{1-R / R^{\prime}}} \frac{z}{R^{\prime}} \phi^{(0)}(x)+\ldots,
$$

as motivated by holographic Higgs models 24. The options now are, to still have only brane Yukawa couplings (despite the fact that the Higgs is a bulk field) or the more natural option of having bulk Yukawa couplings in the up sector, but boundary Yukawas in the down sector (due to the bulk flavor symmetry). We have realized scans with these three options both in the case of MFP and in the standard case in which there is no flavor symmetry. (In this latter case, the three different localization parameters in the RH down sector are computed directly from the mass of the $d, s, b$ quarks.) The result is displayed in Fig. 1, where a random horizontal shift in the points has been introduced to facilitate visualization. In all cases we have fixed $M_{K K}=3 \mathrm{TeV}$. The first three sets of points correspond to RS models with no flavor protection beyond the RS-GIM mechanism. They are, from left to right, for a boundary Higgs, bulk Higgs but boundary Yukawas and bulk Higgs with bulk (up) Yukawas, respectively. The remaining three sets correspond to the same Higgs and Yukawa configurations but with MFP. It is evident from the plot that a value $M_{K K}=3 \mathrm{TeV}$ in the original RS model without flavor protection is ruled out by $\epsilon_{K}$, unless we are willing to make a fine-tuning of a least $\lesssim 10^{-2}$ (corresponding to the ratio of points that survive the bound), independently of where the Higgs and Yukawa couplings live. With MFP, on the other hand, a sizable region $(\sim 17-30 \%)$ of parameter space is allowed by $\Delta F=2$ observables. Furthermore, due to the extra suppression with higher values of $M_{K K}$, if we take $M_{K K}=4 \mathrm{TeV}$, the percentage of points above the bound in models with MFP is about $\sim 40-50 \%$, whereas 


\begin{tabular}{lcc}
\hline \hline \multicolumn{1}{c}{ Model } & \multicolumn{2}{c}{ \% of allowed points } \\
\hline non MFP & $c_{q 3}=0.4$ & $c_{q 3}=0.45$ \\
MFP & 0.4 & 0.4 \\
MFP with BKT $(\mathrm{N}=1)$ & 23.1 & 23.0 \\
MFP with BKT $(\mathrm{N}=2)$ & 11.5 & 14.8 \\
MFP with BKT $(\mathrm{N}=3)$ & 5.9 & 7.8 \\
\hline \hline
\end{tabular}

TABLE I: Ratio of points (in percentage) that are consistent with all relevant $\Delta F=2$ constraints for a boundary Higgs, without MFP, with MFP but no BKT and with MFP and BKT with three different values for the maximun allowed size of the BKT. In these scans we have only included points that satisfy $\operatorname{Max}\left(\left|\tilde{Y}^{u, d}\right|\right) / \operatorname{Min}\left(\left|\tilde{Y}^{u, d}\right|\right) \leq 10$ (see text for details).

it remains at the per cent level in models without MFP. Finally, although we have only displayed the results for the most constraining operator $Q_{4}^{s d}$ in Fig. 1. we have also checked that all other $\Delta F=2$ operators, including those in the up sector, are below current experimental limits [20].

In order to analyze the impact of BKT for the RH down type quarks, we have performed some extra very exhaustive scans for the case of a boundary Higgs. Also, to test the models in a slightly complementary way to the previous scans, we have fixed the parameters in a different way. We have generated random complex $3 \times 3$ Yukawa matrices $\tilde{Y}^{u, d}$, again satisfying $\left|\tilde{Y}^{u, d}\right| \leq 3$. We have then fixed the ratios $f_{q i} / f_{q 3}$ and $f_{-u i} / f_{-u 3}$, with $i=1,2$, from Eq. (18) and checked if the resulting CKM matrix (including the Jarlskog invariant) agrees with the experimentally measured values (better than 30\%). $f_{-u 3}$ and $f_{-d}$ are then fixed by the top and bottom masses, once we choose a value for $f_{q 3}$. In order to test the dependence on $c_{q 3}$ we have done the analysis with two different values, $c_{q 3}=0.4$ and $c_{q 3}=0.45$. In the case that we are not considering MFP, $f_{-d 1}$ and $f_{-d 2}$ have been taken randomly within the ranges suggested by Eqs. (18) and (19). Finally, we have randomly generated diagonal BKTs for the RH down quarks with three different upper bounds, $a_{1,2,3} \leq 3^{2} \delta_{N}, N=1,2,3$, with

$$
\delta_{N} \equiv \frac{N M_{K K} R^{\prime}}{16 \pi^{2}} .
$$

We have done this to estimate the dependence on the actual size of the BKT. As we mentioned, Eq. (38) is nothing but an estimation, as the actual value of the BKTs cannot be computed in the five-dimensional theory. Thus, when we set $N=1$ we do not mean that the cut-off of the model is already at the first KK mode but rather than the NDA estimation might be a bit pesimistic and the actual BKT could turn out to be a bit smaller than expected. Similarly, we have also considered the case that the estimation is accurate and the cut-off is at the third KK mode.

We have generated several thousand points along the previous lines and have analyzed the constraints from all relevant $\Delta F=2$ observables $[20$. The results are summarized in Table I] where we report on the percentage of points that pass all $\Delta F=2$ constraints for each model and size of BKT. These results show a very good consistency with our previous scans in the case that BKTs are neglected (both with and without MFP). In the case of MFP with BKTs, the ratio of points that pass all $\Delta F=2$ constraints is reduced as expected. However, we find that the level of reduction is very sensitive to the size of the BKTs and also somewhat sensitive to the exact localization of the LH top/bottom quarks, $c_{q 3}$. In particular, for $c_{q 3}=0.45$ and $N=1$ (BKT a bit smaller than the NDA estimation), about $15 \%$ of the points are allowed by flavor constraints, getting reduced to $4 \%$ in the case of $c_{q 3}=0.4$ and $N=3$, smaller but still sizably better than the case without MFP. These numbers have been obtained including only those points that do not involve a hierarchy between the different entries of the Yukawa matrices larger than a factor of $10, \operatorname{Max}\left(\left|\tilde{Y}^{u, d}\right|\right) / \operatorname{Min}\left(\left|\tilde{Y}^{u, d}\right|\right) \leq 10$, but we have checked that the results are not very sensitive to that factor. For instance they change by a few per cent up to $\sim 50 \%$ for the case of the models with BKT and $N=3$ if we use only the points with a hierarchy in the Yukawas smaller than 5 (although the statistics is much limited in that case) and barely change if we allow an arbitrary hierarchy.

\section{DISCUSSION}

Recent analyses have shown that flavor observables strongly constrain the parameter space of models with warped extra dimensions and a considerable effort is being put in finding successful flavor symmetries that protect these models from large flavor violations. We have argued that the status of flavor in models with warped extra dimensions is actually not that bad and current experimental limits do not yet require desperate measures. In fact, the built-in flavor protection mechanism present in models with warped extra dimensions, the RS-GIM mechanism, which ensures 
most of the flavor violating observables to remain close to but below current experimental bounds works extremely well. It is only one observable (CP violation in Kaon oscillations) and only due to a very particular, chirality enhanced, contribution that significantly constrains the parameter space in these models. Thus, we have suggested a very simple solution that suppresses the only dangerous set of operators, leaving intact most of the rest of the flavor violating and diagonal structure of the models. In this work, we have shown that the proposal can be easily realized in the quark sector, by imposing a flavor symmetry that enforces a common localization parameter for all the RH quark zero modes in the down sector. We have shown that the contribution to $\Delta F=2$ operators with RH currents (including the chirality enhanced LR contributions) is vanishing at leading (tree level dimension 6 operator) order. We have then computed the most relevant sub-leading (dimension 8 and some loop suppressed) contributions to $\Delta F=2$ processes. A numerical scan shows that, including the contributions from dimension 8 operators, a significant region of parameter space, with a very low $\mathrm{KK}$ scale $\left(M_{K K}=3-4 \mathrm{TeV}\right)$ is compatible with flavor and electroweak precision data. The presence of (loop suppressed) brane kinetic terms, reintroduce FCNC in the RH down sector, thus representing the leading source of flavor violation in the down sector, if they are large. We have performed dedicated scans to analyze the effect of BKTs with the result that they certainly reduce the allowed region of parameter space if they are large. However, we have seen that, if the BKTs are just a bit smaller than the NDA estimate, their effect is not dramatic and we still have sizable regions of parameter space allowed by flavor and electroweak precision tests. In this allowed regions, the hierarchies in the quark masses and mixing angles are still naturally explained through wave function localization. In fact, MFP provides a rationale for the smaller hierarchy in the down sector. This shows that the MFP idea can lead to viable models with warped extra dimensions and new physics accessible at the LHC.

In order to fully study the viability of MFP as a new paradigm in models with warped extra dimensions, some further analyses, some of which are currently under way, are required. First, we have considered the more constraining $\Delta F=2$ observables, including the contribution of the first few KK modes. This gives the main contribution, but a more refined analysis, including the effect of the whole tower of gauge boson and fermion KK modes, which can be done analytically in some cases [12, 13, would be desirable. Also, a detailed analysis of $\Delta F=1$ and electric dipole moment (EDM) contributions can decide which observables are the most likely to give experimental signatures in minimal flavor protection scenarios. $\Delta F=1$ processes were shown in [6], in the absence of flavor protection, to give contributions close to but below current experimental limits, in particular in semileptonic decays of $B$ mesons. Also, the authors of [6] showed that the neutron EDM generates a new strong CP problem, requiring a cancellation of about 5\%. It would be interesting to investigate the situation in the presence of MFP, which, at least for two generations, seems to provide enough suppression of the neutron EDM. Also, we have not explicitly studied models of gauge-Higgs unification. These were shown in [12] to be slightly more constraining than the standard RS model with a fundamental Higgs, due to a kinetic mixing induced by the localized couplings needed to generate non-trivial quark masses and mixings. The resulting bound on $M_{K K}$ turns out to be $\sim 50 \%$ stronger than in the case of a fundamental Higgs. In our case, due to the extra power of $M_{K K}^{-2}$ on the correction, a similar result as the one displayed in Fig. 1 . can be expected for gauge-Higgs unification models with $M_{K K} \sim 4 \mathrm{TeV}$, provided no ultralight modes mix sizably with $d_{R}^{(0) i}$. A detailed analysis, would be however required to fully assess the effectiveness of the MFP paradigm in models of gauge Higgs unification.

Finally, it would be interesting to study how the MFP paradigm can be extended to the leptonic sector. The smaller (about three orders of magnitude) hierarchy of charged lepton masses and the large neutrino mixing angles seem to point to symmetries enforcing universality of some of the localization parameters in the lepton sector. It would be interesting to analyze the interplay of lepton masses and mixing angles and lepton flavor violating processes [25] to try and find the minimal flavor protection scheme in the leptonic sector.

In summary, we have argued that the current experimental situation of models with warped extra dimensions does not require extreme measures yet. Minimal flavor protection on top of the RS-GIM mechanism, together with custodial symmetry and a symmetry protection of the $Z \bar{b}_{L} b_{L}$ coupling seem enough to provide fully realistic models of warped extra dimensions accessible at the LHC. The beauty of minimal flavor protection, beyond its simplicity, is that it still predicts flavor violating processes close to current experimental limits and can therefore be also tested with precision flavor experiments. If these experiments end up ruling out the minimal flavor protection paradigm, less minimal flavor constructions along the lines of [15] would be required.

Note Added: While this work was being completed, Refs. 26] became public. Two different approaches to flavor symmetries in the leptonic sector are discussed in these works. In both works universalities of localization parameters play an important role to generate large neutrino mixing angles and prevent excessive flavor violation along the lines mentioned above. 


\section{Acknowledgments}

We would like to thank K. Agashe, C. Csaki, A. Delgado, A. Falkowski, U. Haisch, M. Neubert, E. Pontón, T. Tait and A. Weiler for useful discussions and comments. Financial support from the Swiss National Science Foundation under contract 200021-117873 is acknowledged. We would also like to thank B. Menor and C. Menor for creating a wonderful working environment during the final stages of this work.

[1] L. Randall and R. Sundrum, Phys. Rev. Lett. 83, 3370 (1999) arXiv:hep-ph/9905221.

[2] Y. Grossman and M. Neubert, Phys. Letti. B 474 (2000) 361 arXiv:hep-ph/9912408.

[3] T. Gherghetta and A. Pomarol, Nucl. Phys. B 586 (2000) 141 arXiv:hep-ph/0003129].

[4] S. J. Huber and Q. Shafi, Phys. Lett. B 498 (2001) 256 arXiv:hep-ph/0010195.

[5] S. J. Huber, Nucl. Phys. B 666 (2003) 269 arXiv:hep-ph/0303183.

[6] K. Agashe, G. Perez and A. Soni, Phys. Rev. D 71 (2005) 016002 arXiv:hep-ph/0408134.

[7] M. S. Carena, E. Ponton, J. Santiago and C. E. M. Wagner, Nucl. Phys. B 759 (2006) 202 arXiv:hep-ph/0607106; Phys. Rev. D 76 (2007) 035006 arXiv:hep-ph/0701055; G. Cacciapaglia, C. Csaki, G. Marandella and J. Terning, Phys. Rev. D 75 (2007) 015003 arXiv:hep-ph/0607146; R. Contino, L. Da Rold and A. Pomarol, Phys. Rev. D 75 (2007) 055014 arXiv:hep-ph/0612048; G. Panico, E. Ponton, J. Santiago and M. Serone, arXiv:0801.1645 [hep-ph].

[8] K. Agashe, A. Delgado, M. J. May and R. Sundrum, JHEP 0308 (2003) 050 arXiv:hep-ph/0308036].

[9] K. Agashe, R. Contino, L. Da Rold and A. Pomarol, Phys. Lett. B 641 (2006) 62 arXiv:hep-ph/0605341].

[10] A. Djouadi, G. Moreau and F. Richard, Nucl. Phys. B 773 (2007) 43 arXiv:hep-ph/0610173;

[11] G. Moreau and J. I. Silva-Marcos, JHEP 0603 (2006) 090 arXiv:hep-ph/0602155.

[12] C. Csaki, A. Falkowski and A. Weiler, arXiv:0804.1954 [hep-ph].

[13] S. Casagrande, F. Goertz, U. Haisch, M. Neubert and T. Pfoh, arXiv:0807.4937 [hep-ph].

[14] S. Davidson, G. Isidori and S. Uhlig, Phys. Lett. B 663 (2008) 73 arXiv:0711.3376 [hep-ph]].

[15] G. Cacciapaglia, C. Csaki, J. Galloway, G. Marandella, J. Terning and A. Weiler, JHEP 0804 (2008) 006 arXiv:0709.1714 [hep-ph]]; A. L. Fitzpatrick, G. Perez and L. Randall, arXiv:0710.1869 [hep-ph]; C. Cheung, A. L. Fitzpatrick and L. Randall, JHEP 0801 (2008) 069 arXiv:0711.4421 [hep-th]]; M. C. Chen and H. B. Yu, arXiv:0804.2503 [hep-ph].

[16] H. Davoudiasl, G. Perez and A. Soni, arXiv:0802.0203 [hep-ph].

[17] K. Agashe, G. Perez and A. Soni, Phys. Rev. Lett. 93 (2004) 201804 arXiv:hep-ph/0406101] K. Agashe, M. Papucci, G. Perez and D. Pirjol, arXiv:hep-ph/0509117

[18] A. Delgado, A. Pomarol and M. Quiros, JHEP 0001 (2000) 030 arXiv:hep-ph/9911252.

[19] H. Georgi, A. K. Grant and G. Hailu, Phys. Lett. B 506 (2001) 207 arXiv:hep-ph/0012379] M. S. Carena, T. M. P. Tait and C. E. M. Wagner, Acta Phys. Polon. B 33 (2002) 2355 arXiv:hep-ph/0207056; H. Davoudiasl, J. L. Hewett and T. G. Rizzo, Phys. Rev. D 68 (2003) 045002 arXiv:hep-ph/0212279; M. S. Carena, E. Ponton, T. M. P. Tait and C. E. M. Wagner, Phys. Rev. D 67 (2003) 096006 arXiv:hep-ph/0212307.

[20] M. Bona et al. [UTfit Collaboration], JHEP 0803 (2008) 049 arXiv:0707.0636 [hep-ph]].

[21] F. del Aguila and J. Santiago, Phys. Lett. B 493 (2000) 175 arXiv:hep-ph/0008143.

[22] F. del Aguila, M. Perez-Victoria and J. Santiago, JHEP 0009 (2000) 011 arXiv:hep-ph/0007316]; Phys. Lett. B 492 (2000) 98 arXiv:hep-ph/0007160.

[23] F. del Aguila, M. Perez-Victoria and J. Santiago, JHEP 0302 (2003) 051 arXiv:hep-th/0302023; JHEP 0610 (2006) 056 arXiv:hep-ph/0601222.

[24] R. Contino, Y. Nomura and A. Pomarol, Nucl. Phys. B 671 (2003) 148 arXiv:hep-ph/0306259.

[25] K. Agashe, A. E. Blechman and F. Petriello, Phys. Rev. D 74 (2006) 053011 arXiv:hep-ph/0606021.

[26] G. Perez and L. Randall, arXiv:0805.4652 [hep-ph]]; C. Csaki, C. Delaunay, C. Grojean and Y. Grossman, arXiv:0806.0356 [hep-ph]. 